\title{
Basosquamous carcinoma: epigenetic considerations in a case
}

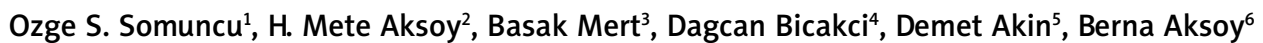

${ }^{1}$ Department of Medical Biology, Faculty of Medicine, Bahçeşehir University, i̇stanbul, Turkey

2Department of Plastic, Reconstructive and Aesthetic Surgery, Faculty of Medicine, Bahçeşehir University, Istanbul, Turkey ${ }^{3}$ School of Medicine, Bahçeşehir University, İstanbul, Turkey

${ }^{4}$ Department of Cardiology, SBU Trabzon Ahi Evren Chest Heart and Vascular Surgery Training and Research Hospital, Trabzon, Turkey ${ }^{5}$ Department of Pharmacology, Faculty of Medicine, Bahçeşehir University, Istanbul, Turkey

${ }^{6}$ Department of Dermatology, Faculty of Medicine, Bahçeşehir University, Istanbul, Turkey

Adv Dermatol Allergol 2020; XXXVII (1): 125-128

DOI: https://doi.org/10.5114/ada.2020.93390

Basosquamous carcinoma (BsC) simulates basal cell carcinoma (BCC) bringing diagnostic difficulties. This infrequent and destructive tumour accounts for $2 \%$ of all non-melanoma skin cancers (NMSCs). Studies disclose the destructive properties of the tumor with an occurrence of distant metastases (almost 7.4\%) greater than that of squamous cell carcinoma (SCC) [1]. As far as we know, there are no reported comparisons for epigenetic/ genetic differences between BsC and healthy cells.

A 68-year-old man presented with a tumoral lesion that slowly enlarged for 6 years and a previous shave biopsy report of BCC. Past medical history revealed diabetes and hypertension with no history of sunburn. Dermatological examination revealed a skin coloured tumoral lesion located on the right ala of the nose measuring $4 \mathrm{~cm}$ at the longest diameter. The patient underwent surgical resection of the tumour reconstructed with a nasolabial flap. The flap is opened up from outer edges, bearing 3-4 $\mathrm{mm}$ of underlying adipose; then, hinged on its bottom, the flap is flipped over medially like a book page. When the flap is sutured to the along defect proximally, the distal flap is graciously rotated $90^{\circ}$ angles and is then folded on itself to create the outer surface of the ala. The histological examination of the surgical specimen revealed $\mathrm{BsC}$ with medium desmoplasia and intense lymphohistiocytic infiltration (Figure 1). A piece of tumor taken from the middle of the tumoral lesion was primarily cultured. Human dermal keratinocytes and fibroblasts were grown in KSFM (keratinocyte serum-free medium) and DMEM (Dulbecco's Modified Eagle Medium) High Glucose medium. Total oxidant status and immunocytochemistry assays for genetic and epigenetic studies were performed in comparison with healthy human der- mal fibroblast (HDF) and human epidermal keratinocyte (HEK) cells. Immunocytochemistry (ICC) analysis was performed to study the effect of genetic/epigenetic diversions on disease formation. The cells were incubated with $2 \%(\mathrm{w} / \mathrm{v})$ paraformaldehyde for $20 \mathrm{~min}$ at $4{ }^{\circ} \mathrm{C}$ for fixation and permeabilized with $0.1 \%$ (v/v) Triton X-100 for 5 min and primed in PBS. The cells were incubated with $2 \%(\mathrm{v} / \mathrm{V})$ goat serum (Sigma, USA) for 10 min at $4^{\circ} \mathrm{C}$ for avoiding non-specific binding of primary antibodies. Samples were again rinsed three times with PBS [2]. They were incubated overnight with primary antibodies at $4^{\circ} \mathrm{C}$. Cells were incubated with rabbit anti-H3AceK36 (1 : 500; Abcam), rabbit anti-H3AceK9 (1: 500; Abcam), rabbit anti-Filaggrin2, rabbit anti-Cytokeratin15, rabbit anti-Phospho-H3(Ser10) overnight at $4^{\circ} \mathrm{C}$. Washed slides were then treated with goat anti-rabbit FITC-conjugated secondary antibodies (1: 100; Abcam). Slides were examined using an Olympus IX51 inverted fluorescence microscope. For total oxidant status analysis, the following protocol was applied. Oxidants existing in the sample oxidize the ferrous molecules into ferric ions. The ferric ions form a coloured complex with xylenol orange in an acidic medium [3]. The colour intensity, measured spectrophotometrically, was related to the total number of oxidant molecules present in the sample. The assay was calibrated with hydrogen peroxide and the results were expressed in terms of micromolar hydrogen peroxide equivalent per litre ( $\mu \mathrm{mol} \mathrm{H}_{2} \mathrm{O}_{2}$ equiv./l).

Expression of actin binding protein fascin differs between some types of skin neoplasia showing infiltrative behaviour of the tumor [4]. Interestingly, we found a massive upregulation of $\beta$-actin expression in $\mathrm{Bs} C$, normal expression levels in HDF and a very low expres-

Address for correspondence: Berna Aksoy Assist. Prof., MD, VM Medicalpark Hospital, Ovacik Discreet, Beside D-100 Highway, No: 36 Basiskele, Kocaeli, Turkey, GSM: 0905326665634, e-mail: bernaaaksoy@gmail.com

Received: 31.08.2018, accepted: 18.10.2018. 

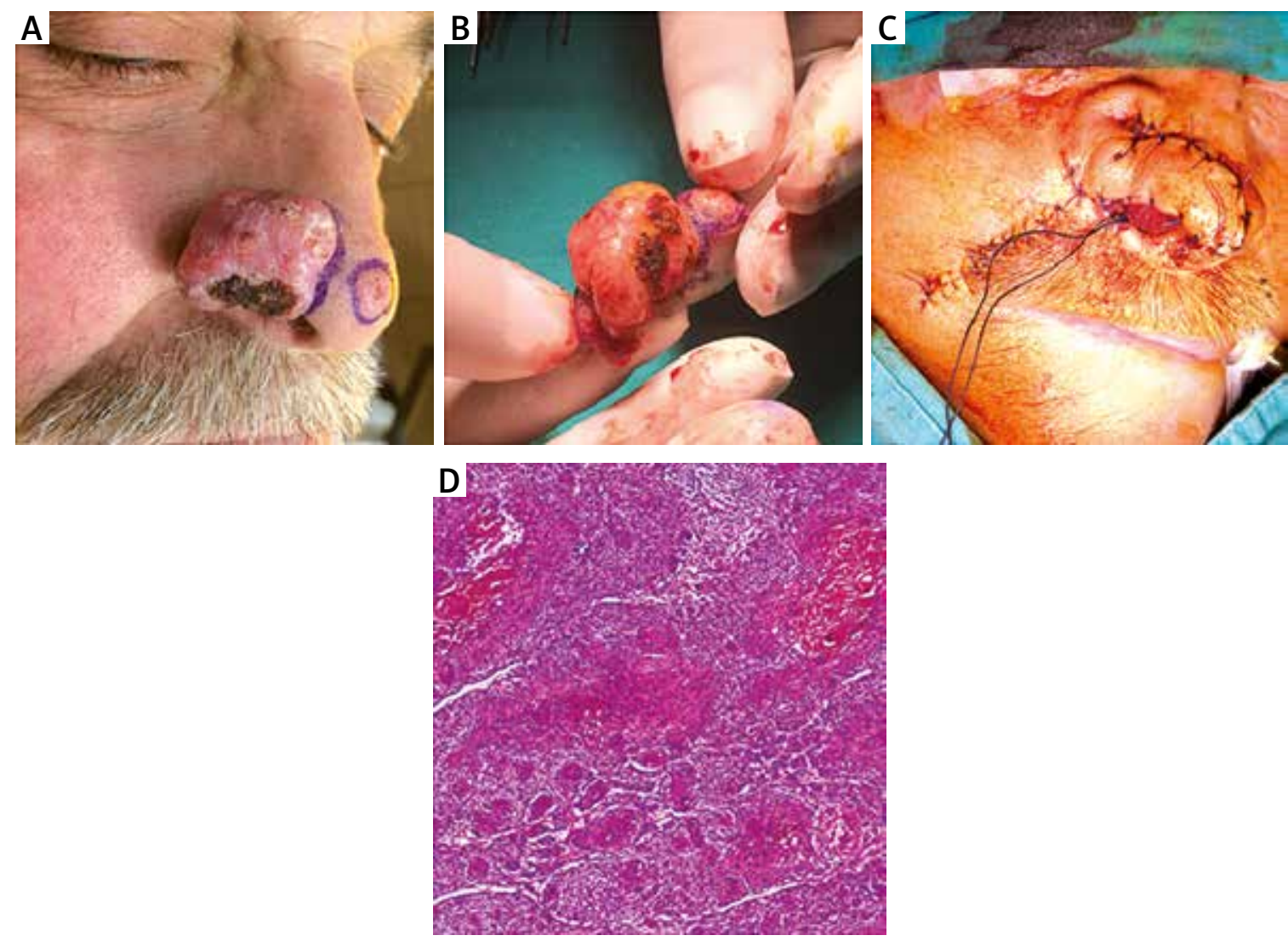

Figure 1. A - Tumoral lesion on the right side of the nose was BsC and at the tip of the nose was BCC. The lesions were seen after en bloc excision (B) and reconstructed via the nasolabial flap (C). D - Histopathological diagnosis was reported as BsC (haematoxylin \& eosin 1000x)

sion in HEK cells (Figure 2 A). Filaggrin 2 has a significant role in epithelial cornification and keratinization [5]. In our research, we found almost no filaggrin 2 expression in BsC cells and the expression was high in healthy HDF and $\mathrm{HEK}$ cells (Figure $2 \mathrm{~B}$ ). Bcl-2 is a family that regulates apoptosis and is responsible for healthy cellular development and prevention of cancer. A higher expression of $\mathrm{BCl}-2$ was observed in $\mathrm{BCC}[6]$. We found higher expressions of $\mathrm{BCl}-2$ in $\mathrm{BsC}$ when compared with HDF and almost similar expressions when compared with HEKs (Figure 2 C). A lower expression of keratin 15 (CK15) was observed on tumours with high invasion rates. The expression of CK15 is downregulated in SCC, while upregulated in BCC [7]. Our results indicated almost two times higher CK15 expression in BsC when compared with healthy HDF cells (Figure 2 D).

Phosphorylation of Histone 3 serine 10 ( $p$-H3S10) is associated with cell cycle progression. H3 S10 phosphorylation leads to the suppression of transformation and it completely blocks methylation of Histone H3 lysine 9 (H3K9) [8]. H3K9 methylation promotes heterochromatin formation. Acetylation of $\mathrm{H} 3 \mathrm{~K} 9$ on the other hand, prevents the methylation. Deacetylation is a precondition for heterochroma- tin formation [8]. In BsC, H3S10 phosphorylation and H3K9 acetylation was found significantly higher than the healthy cells suggesting a lower heterochromatin organization (Figures $2 \mathrm{E}, \mathrm{F}$ ). It correlates with the recent literature suggesting that cancer cells have hypomethylation and hyperacetylation of H3K9 promoting heterochromatin instability leading to increased cellular proliferation [9].

Histone H3 lysine 36 (H3K36) acetylation plays a role in transcriptional activation and regulating a doublestrand break repair pathway, which is associated with cell death and genomic instability [10]. Our data showed no significant difference but an increment of the expression in BsC and HEKs when compared with HDFs suggesting cancer cells may improve their ability to repair DNA breaks and escape from cell death (Figure $2 \mathrm{G}$ ). We also analysed total oxidant status that the cells produce individually. BsC oxidant status was also found almost three times higher than HDFs and greater than that of the standard and HEKs (Figure $2 \mathrm{H}$ ).

Our data confirmed the skin related protein expression differences in BsC. We also showed the histone 3 modification alterations in BsC. The interaction of other histone modifications are still yet to be clarified. 

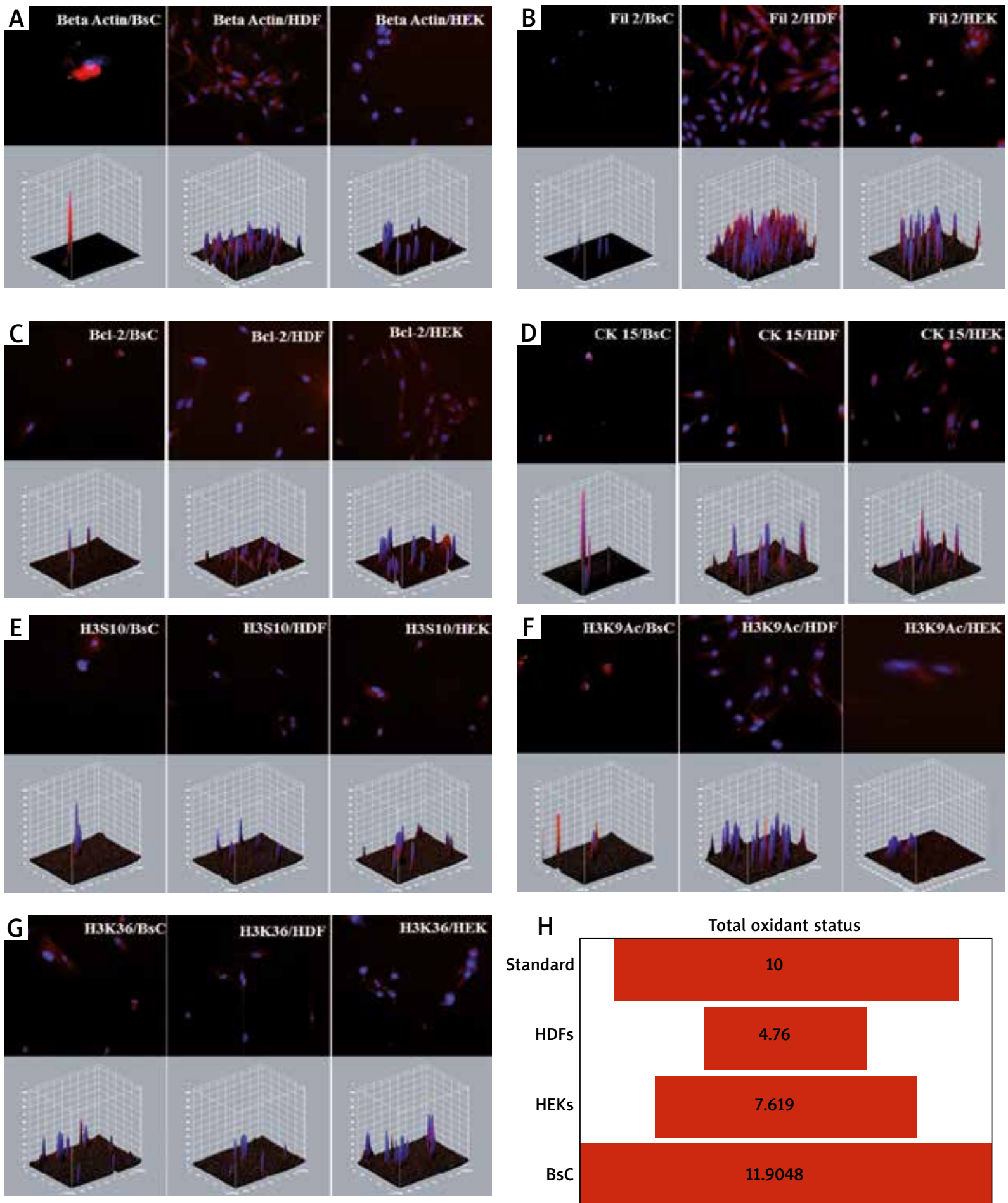

Figure 2. A - Massive upregulation of $\beta$-actin expression in $\mathrm{BsC}$, in comparison with that in HDF and in HEK cells. B - No filaggrin 2 expression in BsC cells and the expression was high in healthy HDF and HEK cells. C - Higher expression of Bcl-2 in BsC when compared with HDF and almost a similar expression in comparison with HEKs. D - Almost two times higher CK15 expression in BsC when compared with healthy HDF and HEK cells. E - Marked H3S10 phosphorylation in BsC in comparison to the healthy cells. F - Higher H3K9 acetylation in BsC in comparison to the healthy cells. G - No significant difference in $\mathrm{H} 3 \mathrm{~K} 36$ acetylation but an increment of the expression in BsC when compared with HDFs. $\mathrm{H}$ - Oxidant status in $\mathrm{BsC}$ is greater than of HDFs, HEKs and the standard 


\section{Acknowledgments}

The authors thank Cuyan Demirkesen for histopathological evaluation.

\section{Conflict of interest}

The authors declare no conflict of interest.

\section{References}

1. Costantino D, Lowe L, Brown DL. Basosquamous carcinoma - an under-recognized, high-risk cutaneous neoplasm: case study and review of the literature. J Plast Reconst Aesth Surg 2006; 59: 424-8.

2. Ma P, Schultz RM. Histone deacetylase 1 (HDAC1) regulates histone acetylation, development, and gene expression in preimplantation mouse embryos. Dev Biol 2008; 319: 110-20.

3. Erel O. A new automated colorimetric method for measuring total oxidant status. Clin Biochem 2005; 38: 1103-11.

4. Goncharuk VN, Ross JS, Carlson JA. Actin binding protein fascin expression in skin neoplasia. J Cutan Pathol 2002; 29: 430-8.

5. Ross-Hansen K, Østergaard O, Tanassi JT, et al. Filaggrin is a predominant member of the denaturation-resistant nickel-binding proteome of human epidermis. J Invest Dermatol 2014; 134: 1164-6.

6. Smoller BR, Rijn M, Lebrun D, Warnke RA. bcl-2 expression reliably distinguishes trichoepitheliomas from basal cell carcinomas. Br J Dermatol 1994; 131: 28-31.

7. Song IY, Balmain A. Cellular reprogramming in skin cancer. Semin Cancer Biol 2015; 32: 32-9.

8. Equilibrina I, Krayukhina E, Inoue K, et al. The role of phosphorylation of histone $\mathrm{H} 3$ at serine 10 in chromatin condensation in vitro. Chromosome Science 2015; 18: 9-14.

9. Hosseini A, Minucci S. A comprehensive review of lysinespecific demethylase 1 and its roles in cancer. Epigenomics 2017; 9: 1123-42.

10. Pai CC, Deegan RS, Subramanian L, et al. A histone H3K36 chromatin switch coordinates DNA double-strand break repair pathway choice. Nat Commun 2014; 5: 4091. 